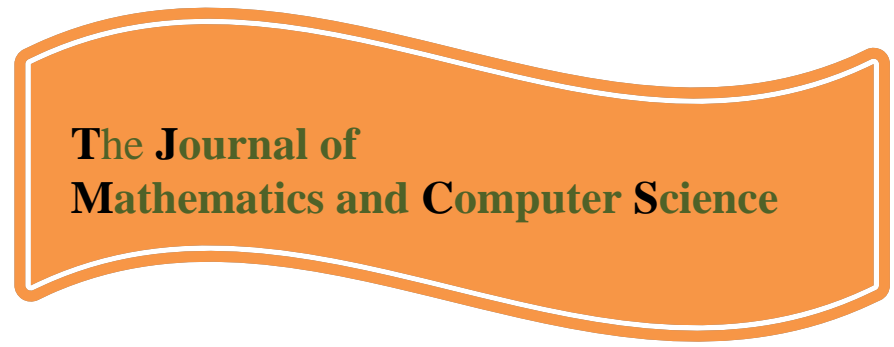

Available online at

http://www.TJMCS.com

The Journal of Mathematics and Computer Science Vol .5 No.2 (2012) 97-104

\title{
Efficient Analytical Approaches for Motion of a Spherical Solid Particle in Plane Couette Fluid Flow using Nonlinear Methods
}

\author{
S.E.Ghasemi1 ${ }^{1}$ S.Jalili Palandi , M.Hatami , D.D.Ganji \\ Department of Mechanical Engineering, Babol University of Technology, Babol, Iran
}

Received: February 2012, Revised: November 2012

Online Publication: December 2012

\begin{abstract}
In this study, we approach a spherical particle in plane Couette fluid flow problem utilizing Adomian's decomposition method (ADM) as well as variational iteration method (VIM) to find a rapidly convergent power series solution. Equation of particle's motion in Couette flow considering the rotation and shear effects on lift force and neglecting gravity has been investigated by Vander Werff. The required time and distance for a spherical particle to reach terminal velocity trajectory of particle obtained which has application in transferring the medicine in blood in medical area or control of particles motion during spraying or injecting processes in industry. The precious contribution of the work is introducing a new fast and efficient solution of analytical methods in a spherical particle in plane Couette fluid flow over the previous numerical and analytical counterpart results in literature, while it is shown that both methods give approximations of a high degree of accuracy and least computational effort for studying particle motion in Couette fluid flow.
\end{abstract}

Keywords: Spherical particle,Couette flow,Adomian's decomposition method,variational iteration method.

\section{Introduction.}

Numerical solutions of equation derived from the balance equation of external forces applied to a particle in interaction with drag, virtual mass force, the Basset force, lift force, etc. have been broadly considered in literature; albeit an exact analytical expression that gives a better understanding of the physical behavior of the sphere-fluid combination and the accelerated motion of the sphere is still worth studying. Knowing that, an analytical solution is usually the more

\footnotetext{
${ }^{1}$ Corresponding author: Tel/Fax: +98 1113234205

E-mail address: s.ebrahim.ghasemi@gmail.com
} 
preferred and convenient method in engineering area because of less computational work as well as high accuracy. The cases that particles come into contact with a solid or fluid boundary and obtaining the drag coefficient in order to predict the free falling velocity of particles have been immense interest of many researchers. The main objective of these problems is to estimate the time and distance required to reach terminal velocity for a given sphere-fluid combination prior to making the reliable determination of the sphere settling velocity. Regarding the aforementioned reasons the study of the accelerated motion of a sphere particle in a plane Couette fluid using analytical ADM and VIM and comparing the agreement and even improvement of obtained results in comparison with previous analytical and numerical solutions and determines the most suited approach is focused in current study.

Jalal et al. solved a spherical particle's motion in Couette flow using Homotopy perturbation method (HPM) and got comparable results to numerical ones [1]. A combined experimentalnumerical study was performed for a drag coefficient-Reynolds correlation for a single spherical particle rolling down a smooth plane for three classified regimes of Re from low up to $10^{5}$, in an incompressible Newtonian media by Jan and Chen [2]. Explicit equations of correlation between Reynolds and drag coefficients proposed by Yow et al. for different shapes of particles including spheres, cube octahedrons, octahedrons, cubes, tetrahedrons, discs, cylinders and rectangular parallelepipeds [3]. The unsteady rolling motion of a spherical particle restricted to a tube was studied analytically by Jalal and Ganji. An exact solution of particle velocity and acceleration motion under some practical conditions was obtained through applying HPM [4]. Jalal and Ganji proposed an analytically solution for acceleration motion of a spherical particle rolling down an inclined boundary with drag coefficient which is correlated linearly to Re in a specific range using HPM. Various inclination angles were studied and observed that settling velocity, acceleration duration and displacement are proportional to amount of inclination angle while for a constant inclination angle; settling velocity and acceleration duration are decreased by increasing the fluid viscosity [5]. Motivated by the above mentioned works, the main objective of the present research paper is to see the effect and accuracy of ADM and VIM methods on the 2D motion of a spherical particle in plane Couette fluid flow that has already been solved by numerical method. Results show that both methods have high degree of accuracy, and they are faster and more efficient than the previous method.

\section{Statement of Problem}

The early equation of motion of sphere particles' lift effect in a Couette flow has been studied by Steverding [6]. In that study the lift force of particle was assumed due to merely rotation of particle, while later Saffman proved that the contribution of shear effect in particle's motion is not less important than the former one [7]. Vander Werff proposed a more comprehensive equation of motion of sphere particle in Couette flow which covers the shortfall of previous studies [8].

The Vander Werff model for particle motion in Couette flow is adopted in this study while the positive direction rotation of particle is clockwise and combined effects of inertia, gravity and buoyancy are assumed negligible. So, the inertia force in left hand of force balance equation is the product of mass of sphere particle by its acceleration $(\ddot{x}, \ddot{y}, 0)$ :

$T=\frac{4 \pi a^{3}}{3} \rho_{s} \dot{V}=\frac{4 \pi a^{3}}{3} \rho_{s}(\ddot{x}, \ddot{y}, 0)$

Where $a, \rho$ and $V$ are the radius, density and velocity of sphere particle respectively. $\dot{V}$ is first derivative of particle's velocity and $\ddot{x}$ and $\ddot{y}$ are second derivatives of particles' motion in horizontal and vertical directions respect to time.

In order to calculate the drag force the velocities of the sphere particle are considered small adequately so that the Stokes law can be governed:

$T_{D x}=6 \pi \mu a V_{r x}=6 \pi \mu a(\dot{x}-\alpha y)$

$T_{D y}=6 \pi \mu a V_{r y}=6 \pi \mu a \dot{y}$

While $\mu$ signify the viscosity of fluid.

The rotation and shear portion of the particle's lift force is obtained as: 


$$
\begin{aligned}
T_{R x} & =\frac{1}{2} \pi a^{3} \rho \alpha \dot{y} \\
T_{R y} & =\frac{1}{2} \pi a^{3} \rho \alpha(\alpha y-\dot{x}) \\
T_{S x} & =0 \\
T_{S y} & =6.46 a^{2} \rho \alpha^{1 / 2} v^{1 / 2}(\alpha y-\dot{x})
\end{aligned}
$$

Where $\alpha$ is defined as positive proportionality constant.

An illustration of the spherical particle in plane Couette fluid flow and exerted forces on particle are shown in Fig.1. The mass of particle is assumed in the center of sphere and the forces caused from the rotation and shear fields and their interactions on drag and lift forces of particle are illustrated in Figs. 1.a and 1.b respectively. By forming the force balance equation of the inertia force to the drag and lift forces; the equations of motion for the particle are driven as:

$T=T_{R}+T_{S}-T_{D}$

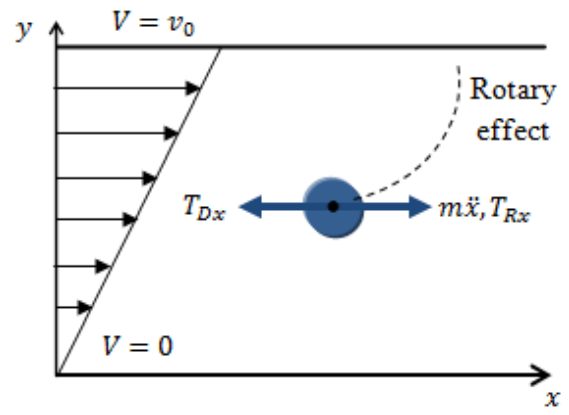

a. Drag interactions

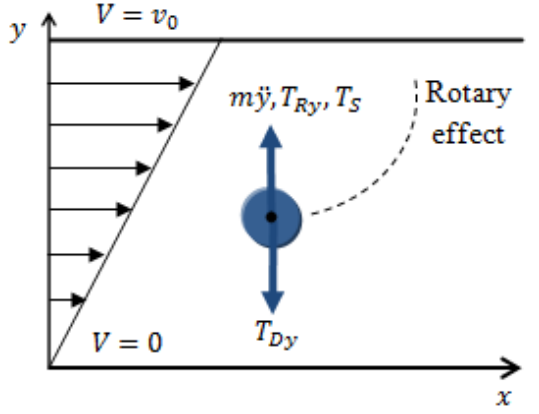

b. Lift interactions

\section{Figure 1. Schematic view of exerted forces on a spherical particle in couette fluid flow}

Eventually, by substituting the Eqs. (2) and (4) into Eq. (5) the system of equation of motion of sphere particle in plane Couette flow yields:

$$
\begin{aligned}
& \frac{4 \pi a^{3}}{3} \rho_{s} \ddot{x}=\frac{1}{2} \pi a^{3} \rho \alpha \dot{y}-6 \pi \mu a(\dot{x}-\alpha y) \\
& \frac{4 \pi a^{3}}{3} \rho_{s} \ddot{y}=\left(\frac{1}{2} \pi a^{3} \rho \alpha+6.46 a^{2} \rho \alpha^{1 / 2} v^{1 / 2}\right)(\alpha y-\dot{x})-6 \pi \mu a \dot{y} \\
& \begin{cases}\frac{4 \pi a^{3}}{3} \rho_{s} \ddot{x} & =\frac{1}{2} \pi a^{3} \rho \alpha \dot{y}-6 \pi \mu a(\dot{x}-\alpha y) \\
\frac{4 \pi a^{3}}{3} \rho_{s} \ddot{y} & =\left(\frac{1}{2} \pi a^{3} \rho \alpha+6.46 a^{2} \rho \alpha^{1 / 2} v^{1 / 2}\right)(\alpha y-\dot{x})-6 \pi \mu a \dot{y}\end{cases}
\end{aligned}
$$

For simplicity the governing equations have been expressed as:

$$
\left\{\begin{array}{l}
\ddot{x}=A \dot{y}+B(\dot{x}-\alpha y) \\
\ddot{y}=B \dot{y}-(A+C)(\dot{x}-\alpha y)
\end{array}\right.
$$

Where the coefficients A-C are defined as:

$$
\begin{aligned}
& A=\left(\frac{3 \alpha}{8}\right)\left(\frac{\rho}{\rho_{S}}\right) \\
& B=-\left(\frac{9 v}{2 r^{2}}\right)\left(\frac{\rho}{\rho_{S}}\right) \\
& C=1.542\left(\frac{\sqrt{\alpha \nu}}{r}\right)\left(\frac{\rho}{\rho_{S}}\right)
\end{aligned}
$$

An appropriate initial condition is required in order to avoid trapping the procedure in nontrivial solution:

$x(t=0)=0, \dot{x}(t=0)=u_{0}$ 
$y(t=0)=0, \dot{y}(t=0)=v_{0}$

Since, the procedure of solving the Eq.8 is autonomous of constants A, B and C; so, for generalization and simplyfication of problem for future cases with different physical conditions the constatns which represent physical properties are assumed to be:

$A=B=C=1$

$u_{0}=v_{0}=1$

\section{Results and Discussions}

\subsection{Implementation of Adomian decomposition method for current problem}

ADM applied for solving the Eq. (8) with assumptions (12) and initial conditions (13):

$$
\begin{aligned}
& X_{n+1}(t)=\int_{0}^{t} \int_{0}^{t}\left\{-\left(\frac{d}{d t} X_{n}(t)\right)+\frac{d}{d t} Y_{n}(t)+Y_{n}(t)\right\} d t d t \\
& Y_{n+1}(t)=\int_{0}^{t} \int_{0}^{t}\left\{-\left(\frac{d}{d t} Y_{n}(t)\right)-\frac{d}{d t} X_{n}(t)+Y_{n}(t)\right\} d t d t \\
& X_{0}(t)=t \\
& Y_{0}(t)=t \\
& X_{1}(t)=\frac{1}{6} t^{3} \\
& Y_{1}(t)=-t^{2}+\frac{1}{6} t^{3} \\
& X_{2}(t)=-\frac{1}{12} t^{4}-\frac{1}{3} t^{3}+\frac{1}{120} t^{5} \\
& Y_{2}(t)=\frac{1}{3} t^{3}-\frac{1}{6} t^{4}+\frac{1}{120} t^{5} \\
& X_{3}(t)=\frac{1}{6} t^{4}-\frac{1}{180} t^{6}+\frac{1}{5040} t^{7} \\
& Y_{3}(t)=\frac{1}{15} t^{5}-\frac{1}{120} t^{6}+\frac{1}{5040} t^{7} \\
& X=\sum_{i=0}^{n} X_{i}(t) \\
& Y=\sum_{i=0}^{n} Y_{i}(t) \\
& v_{x}(t)=\frac{d}{d t} x \\
& v_{y}(t)=\frac{d}{d t} y
\end{aligned}
$$



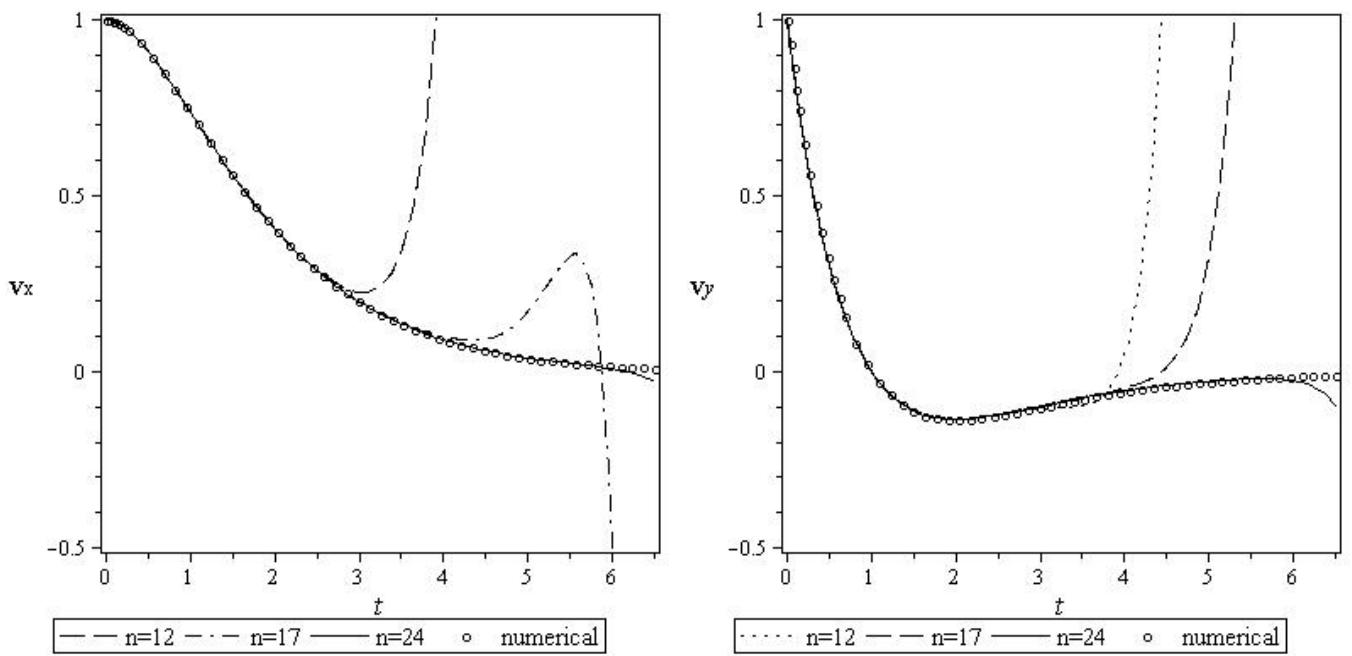

Figure 2. ADM approximant and numerical solutions of Eqs. (8)

Table 1. The results of ADM and fourth order Runge-Kutta method for $v_{x}$

\begin{tabular}{cccccccc}
\hline $\mathrm{t}(\mathrm{s})$ & $\boldsymbol{v}_{\boldsymbol{x} \text { (numerical })}(\boldsymbol{t})$ & $\begin{array}{c}\boldsymbol{v}_{\boldsymbol{x}(\mathrm{ADM})}(\boldsymbol{t}) \\
\mathrm{n}=12\end{array}$ & $\begin{array}{c}\boldsymbol{v}_{\boldsymbol{x}(\mathrm{ADM})}(\boldsymbol{t}) \\
\mathrm{n}=17\end{array}$ & $\begin{array}{c}\boldsymbol{v}_{\boldsymbol{x}(\mathrm{ADM})}(\boldsymbol{t}) \\
\mathrm{n}=24\end{array}$ & $\begin{array}{c}\text { Error }_{A D M} \\
\mathrm{n}=12\end{array}$ & $\begin{array}{c}\text { Error }_{A D M} \\
\mathrm{n}=17\end{array}$ & $\begin{array}{c}\text { Error }_{A D M} \\
\mathrm{n}=24\end{array}$ \\
0 & 1 & 1 & 1 & 1 & 0 & 0 & 0 \\
1 & 0.735759 & 0.735759 & 0.909796 & 0.735759 & $2.04 \mathrm{E}-08$ & $2.78 \mathrm{E}-08$ & $2.777 \mathrm{E}-08$ \\
2 & 0.406006 & 0.406111 & 0.735759 & 0.406006 & 0.000105 & $2.29 \mathrm{E}-09$ & $2.498 \mathrm{E}-08$ \\
3 & 0.199148 & 0.224893 & 0.557825 & 0.199148 & 0.025745 & $3.54 \mathrm{E}-05$ & $2.351 \mathrm{E}-08$ \\
4 & 0.091578 & 1.248554 & 0.406006 & 0.091578 & 1.156976 & 0.004553 & $5.524 \mathrm{E}-07$ \\
5 & 0.040428 & 19.84095 & 0.287299 & 0.040307 & 19.80052 & 0.124557 & 0.000121 \\
6 & 0.017351 & 174.7112 & 0.199184 & 0.009652 & 174.6938 & 0.465109 & 0.007701 \\
\hline
\end{tabular}

Table 2. The results of ADM and fourth order Runge-Kutta method for $\boldsymbol{v}_{\boldsymbol{y}}$

\begin{tabular}{cccccccc}
\hline $\mathrm{t}(\mathrm{s})$ & $\boldsymbol{v}_{\boldsymbol{y}(\text { numerical })}(\boldsymbol{t})$ & $\begin{array}{c}\boldsymbol{v}_{\boldsymbol{y}(\boldsymbol{A D M})}(\boldsymbol{t}) \\
\mathrm{n}=12\end{array}$ & $\begin{array}{c}\boldsymbol{v}_{\boldsymbol{y}(\boldsymbol{A D M})}(\boldsymbol{t}) \\
\mathrm{n}=17\end{array}$ & $\begin{array}{c}\boldsymbol{v}_{\boldsymbol{y}(\boldsymbol{A D M})}(\boldsymbol{t}) \\
\mathrm{n}=24\end{array}$ & $\begin{array}{c}\boldsymbol{E r r o r}_{\boldsymbol{A D M}} \\
\mathrm{n}=12\end{array}$ & $\begin{array}{c}\boldsymbol{E r r o r}_{\boldsymbol{A D M} M} \\
\mathrm{n}=17\end{array}$ & $\begin{array}{c}\boldsymbol{E r r o r}_{\boldsymbol{A D M}} \\
\mathrm{n}=24\end{array}$ \\
0 & 1 & 1 & 1 & 1 & 0 & 0 & 0 \\
1 & $-4.12 \mathrm{E}-08$ & $-1.70 \mathrm{E}-08$ & $-4.45 \mathrm{E}-14$ & $-4.52 \mathrm{E}-15$ & $2.45 \mathrm{E}-08$ & $4.14 \mathrm{E}-08$ & $4.14 \mathrm{E}-08$ \\
2 & -0.135343 & -0.135433 & -0.135335 & -0.135343 & $9.37 \mathrm{E}-05$ & $4.36 \mathrm{E}-08$ & $4.11 \mathrm{E}-08$ \\
3 & $-9.96 \mathrm{E}-02$ & -0.107613 & -0.099556 & -0.099572 & 0.008026 & $1.85 \mathrm{E}-05$ & $4.53 \mathrm{E}-08$ \\
4 & $-5.49 \mathrm{E}-02$ & 0.032745 & -0.049852 & -0.054952 & 0.087692 & 0.005097 & $7.07 \mathrm{E}-08$ \\
5 & $-2.70 \mathrm{E}-02$ & 8.308031 & 0.305092 & -0.027031 & 8.334983 & 0.332043 & $8.09 \mathrm{E}-05$ \\
6 & $-1.24 \mathrm{E}-02$ & 143.6022 & 8.694908 & -0.023423 & 143.6146 & 8.707302 & 0.011024 \\
\hline
\end{tabular}

Table 3. MSE of ADM for various series terms

\begin{tabular}{ccccccc}
\hline & $\boldsymbol{v}_{\boldsymbol{x}(\boldsymbol{V I M})}(\boldsymbol{t})$ & $\boldsymbol{v}_{\boldsymbol{x}(\boldsymbol{V I I})}(\boldsymbol{t})$ & $\boldsymbol{v}_{\boldsymbol{x}(\boldsymbol{V I M})}(\boldsymbol{t})$ & $\boldsymbol{v}_{\boldsymbol{y}(\boldsymbol{V I M})}(\boldsymbol{t})$ & $\boldsymbol{v}_{\boldsymbol{y}(\boldsymbol{V I M})}(\boldsymbol{t})$ & $\boldsymbol{v}_{\boldsymbol{y}(\boldsymbol{V I I})}(\boldsymbol{t})$ \\
& $\mathrm{n}=12$ & $\mathrm{n}=17$ & $\mathrm{n}=24$ & $\mathrm{n}=12$ & $\mathrm{n}=17$ & $\mathrm{n}=24$ \\
MSE & 4415.903 & $3.312 \mathrm{E}-02$ & $8.472 \mathrm{E}-06$ & 2956.376 & 10.8471 & $1.736 \mathrm{E}-05$ \\
\hline
\end{tabular}

Eq. (14) continued up to $n=24$ to get an exact solution with reasonable computationally effort. The 2D profiles of velocity of particle are depicted in Fig.2. It can be easily deduced in Fig.2 that the results obtained from $\mathrm{ADM}$ for $n=24$ are almost same as the numerical result in specified time duration. Furthermore, for more understanding of ADM operation the approximant values of ADM results for $n=12,17$ and 24 as well as the error computed from numerical results inserted in table 1 , 2. From the table 3 which shows the mean square error (MSE) for different series terms involved of ADM; it is found that the velocities of particle in both $x$ and $y$ directions for $n=24$ has the least amount of MSE and hence cover the moving particle velocities for longer time. 


\subsection{Implementation of variation iteration method for current problem}

The solution of Eq. (8) using VIM, assumptions (12) and initial condition (13) is obtained as:

$$
\begin{aligned}
& \frac{d^{2}}{d t^{2}} x(t)+\frac{d}{d t} x(t)-\left(\frac{d}{d t} y(t)\right)-y(t)=0 \\
& \frac{d^{2}}{d t^{2}} y(t)+\frac{d}{d t} y(t)+\frac{d}{d t} x(t)-y(t)=0 \\
& x_{n+1}(t)=x_{n}(t)+\int_{0}^{t} \lambda_{1}\left(\frac{d^{2}}{d \tau^{2}} x_{n}(\tau)+\frac{d}{d \tau} x_{n}(\tau)-\left(\frac{d}{d \tau} y_{n}(\tau)\right)-y_{n}(\tau)\right) d \tau
\end{aligned}
$$

$y_{n+1}(t)=y_{n}(t)+\int_{0}^{t} \lambda_{2}\left(\frac{d^{2}}{d \tau^{2}} y_{n}(\tau)+\frac{d}{d \tau} y_{n}(\tau)+\left(\frac{d}{d \tau} x_{n}(\tau)\right)-y_{n}(\tau)\right) d \tau$

(23b)

$$
\begin{aligned}
& \lambda_{1}=e^{\tau-t}-1 \\
& \lambda_{2}=e^{\tau-t}-1 \\
& x_{0}(t)=1-e^{-t} \\
& y_{0}(t)=1-e^{-t} \\
& x_{1}(t)=t \\
& y_{1}(t)=-2+2 e^{-t}+2 t e^{-t}+t \\
& x_{2}(t)=-2 t-5 e^{-t}+5-2 t e^{-t}+\frac{1}{2} t^{2} \\
& y_{2}(t)=9-9 e^{-t}-4 t e^{-t}-4 t+\frac{1}{2} t^{2}-t^{2} e^{-t} \\
& x_{3}(t)=9 t-14 e^{-t}-14+6 t e^{-t}-2 t^{2}+\frac{1}{6} t^{3}+t^{2} e^{-t} \\
& y_{3}(t)=-36+36 e^{-t}+20 t e^{-t}+17 t-3 t^{2}+4 t^{2} e^{-t}+\frac{1}{3} t^{3} e^{-t}+\frac{1}{6} t^{3}
\end{aligned}
$$

$$
\begin{aligned}
& v_{x}(t)=\frac{d}{d t} x_{n}(t) \\
& v_{y}(t)=\frac{d}{d t} y_{n}(t)
\end{aligned}
$$
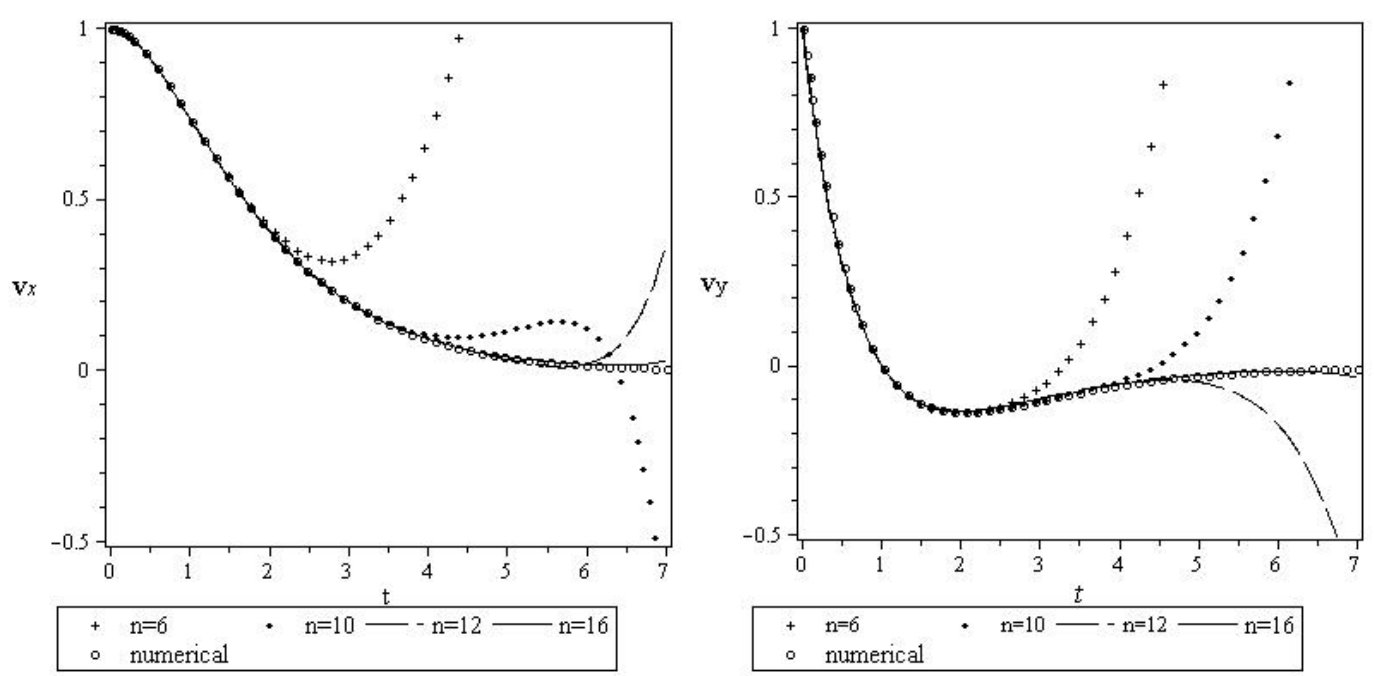
S.E.Ghasemi , S.Jalili Palandi , M.Hatami , D.D.Ganji / TJ MCS Vol .5 No. 2 (2012) 97-104

Figure 3. VIM approximant and numerical solutions of Eqs. (8)

Table 4. The results of VIM and fourth order Runge-Kutta method for $v_{x}$

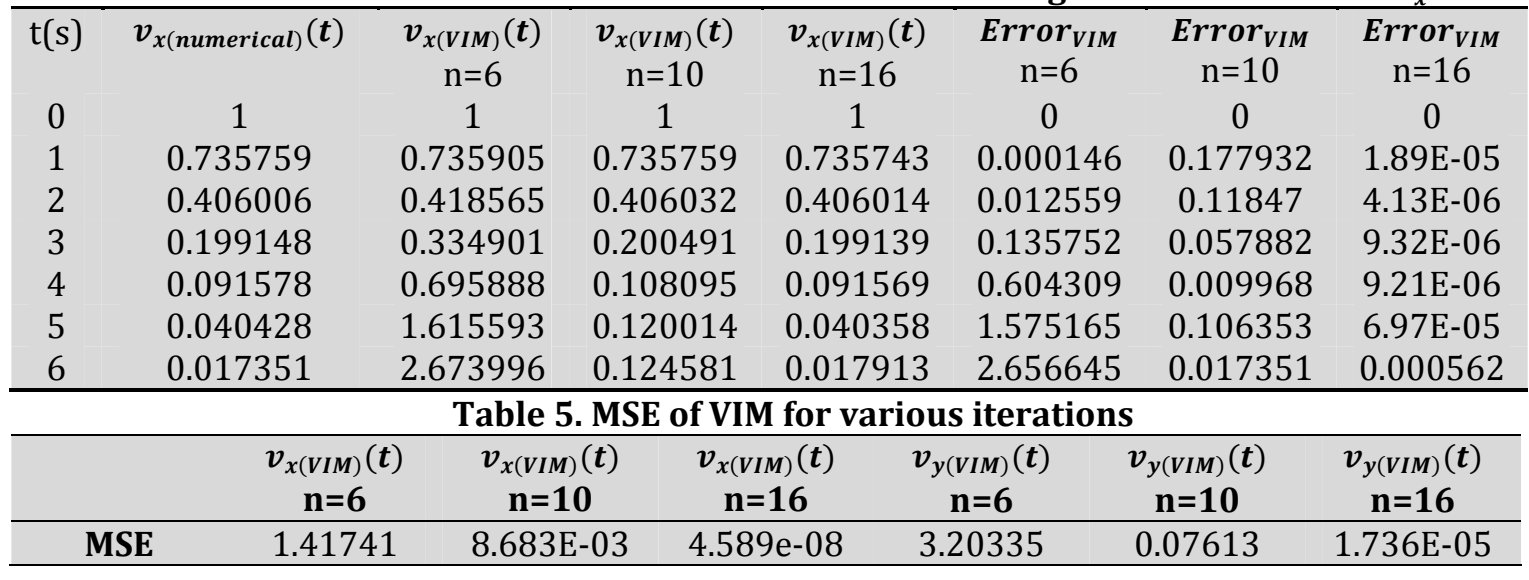

In other stage VIM was applied in order to obtain exact solution of Eq. (8). The results of Eq.33 obtained for four instantaneous counters of $n$ up to $n=16$ which the acceptable results of equation (8) achieved. The variation of velocities of particle in both horizontal and vertical motion versus time are illustrated in Fig. 3 in which the terminal velocity of particle approaches to exact value as the number of iterations increase. The numerical solution is referred as exact value and the calculated values of $v_{x}$ in various time instances are compared with exact values and the errors are tabulated in table 4. From computed mean square error (MSE) values in table 5 it can be concluded that the 2D profiles of particle velocities has the minimum divergence from the exact solution for $n=16$.

\section{Conclusion}

A new approach for analytically handling a couple of equations of a spherical particle motion in a plane Couette fluid flow has been presented. The author assumed a 2D velocity profile of incompressible Newtonian flow in horizontal and vertical motions as the result of solutions. ADM and VIM have been applied and the results compared to previous numerical results. It was observed that clearly by adding to series terms in both methods the moving particle is covered for longer. In this particular case study observed that the considered spherical particle gets to steady state of motion in approximately $6 \mathrm{~s}$ after releasing in the Couette fluid flow. However, making a trade off between accuracy, computational burden and efficiency got the author to choose reasonable amount of series terms that not only obtain an exact solution for present equation but also, propose a faster and computationally more efficient approach for dealing with this sort of problems rather the previous numerical and analytical method.

\section{References.}

[1] M. Jalaal, M.G. Nejad, P. Jalili, M. Esmaeilpour, H. Bararnia, E. Ghasemi, S. Soleimani, D.D. Ganji, S.M. Moghimi," Homotopy perturbation method for motion of a spherical solid particle in plane Couette fluid flow", Comput. And Math. With Appl. 61 (2011) 2267-2270.

[2] D. Jan, J.C. Chen, "Movements of a sphere rolling down an inclined plane", J.Hydraulic Res. 35 (5) (1997) 689-706.

[3] H.N. Yow, M.J. Pitt, A.D. Salman, "Drag correlations for particles of regular shape", Advanced Powder Technol. 16(4) (2005) 363-372.

[4] M. Jalaal, D.D. Ganji, "On unsteady rolling motion of spheres in inclined tubes filled with incompressible Newtonian fluids", Advanced Powder Technol. 22 (2011) 58-67. 
[5] M. Jalaal, D.D. Ganji," An analytical study on motion of a sphere rolling down an inclined plane submerged in a Newtonian fluid", Powder Technol. 198 (2010) 82-92.

[6] B. Steverding, "Critical Reynolds Numbers for Flows Containing Particles", Z. angew. Math. Phys. 79 (1968) 513.

[7] P.G. Saffman, "The Lift on a Small Sphere in a Slow Shear Flow", J. Fluid Mech. 22 (1965) 385.

[8] T.J. VanderWerff, "Critical Reynolds number for a spherical particle in plane Couette flow", Zeitschrift für Angewandte Mathematik und Physik. 21 (1970) 825-830. 